\title{
Development of Morphologically Discrete PEG-PDLLA Nanotubes for Precision Nanomedicine
}

Annelies C. Wauters, ${ }^{\dagger \dagger}$ Imke A. B. Pijpers, ${ }^{\dagger}$ Alexander F. Mason, ${ }^{\dagger} \odot$ David S. Williams, ${ }^{\S}$ Jurjen Tel, ${ }^{\dagger}$ Loai K. E. A. Abdelmohsen, ${ }^{*}{ }^{\dagger}$ and Jan C.M. van Hest* ${ }^{*} \dagger$

${ }^{\dagger}$ Bio-Organic Chemistry, Institute for Complex Molecular Systems, Eindhoven University of Technology, P.O. Box 513 (STO 3.41), 5600 MB Eindhoven, The Netherlands

${ }^{\ddagger}$ Department of Biomedical Engineering, Laboratory of Immunoengineering, Eindhoven University of Technology, $5600 \mathrm{MB}$ Eindhoven, The Netherlands

${ }^{\S}$ Department of Chemistry, College of Science, Swansea University, Swansea, United Kingdom

Supporting Information

ABSTRACT: Precise control over the morphological features of nanoparticles is an important requisite for their application in nanomedical research. Parameters such as size and shape have been identified as critical features for effective nanotherapeutic technologies due to their role in circulation, distribution, and internalization in vivo. Tubular PEGPDLLA polymersomes (nanotubes) exhibit an interesting morphology with potential for immunotherapeutics, as the elongated shape can affect cell-particle interactions. Developing methodologies that permit control over the precise form of such nanotubes is important for their biomedical implementation due to the stringent physicochemical constraints for efficacious performance. Through careful control over the engineering process, we demonstrate the generation of well-defined nanotubes based on polymersomes as small as 250 and 100 $\mathrm{nm}$, which can be successfully shape transformed. The quality of the resulting nanostructures was established by physical characterization using AF4-MALS and cryo-TEM. Moreover, we show the successful loading of such nanotubes with model payloads (proteins and drugs). These findings provide a promising platform for implementation in biomedical applications in which discrete structure and functionality are essential features.

\section{INTRODUCTION}

The development of therapeutic nanoparticles has progressed beyond its early phase, during which challenges such as drug solubilization and delivery were paramount to consider more complex issues associated with morphology and specific interactions with biological interfaces. ${ }^{1-4}$ The physicochemical properties of nanotherapeutic technologies, including size, shape, composition, and surface chemistry, are receiving greater attention as the key determinants of efficacy and safety due to the influence of these parameters on blood circulation, vascular penetration, biodistribution, cellular uptake, and immunogenicity. ${ }^{5-10}$

Having identified that nanoparticles with a size $\sim 100 \mathrm{~nm}$ demonstrate improved performance in terms of circulation times and cellular uptake, the importance of size is a wellestablished design parameter for nanomedicine. ${ }^{1,11,12}$ More recently, the effect of shape has fueled research for its potential to further improve the efficacy of nanoparticles. ${ }^{13-17}$ For example, it has been demonstrated that nature-inspired discoidal or tubular nanoparticles-having a comparable morphology to disk-shaped red blood cells and rod-shaped bacteria-are less susceptible to phagocytosis. ${ }^{10}$ This morphologically induced stealth-like property was shown to reduce

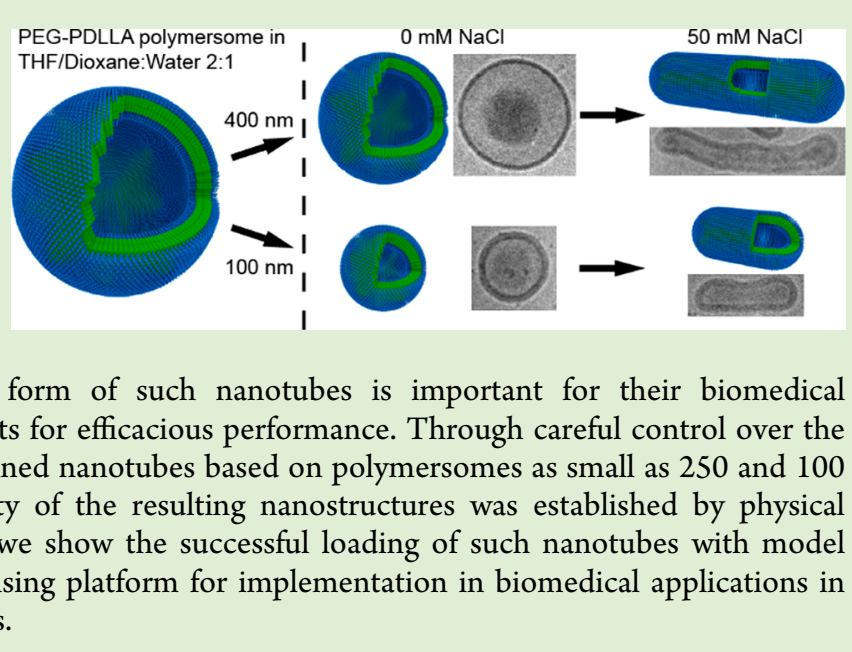

adverse cardiopulmonary distress by avoiding clearance of the intravenously administrated nanoparticles by pulmonary intravascular macrophages (PIMs). ${ }^{5}$ Worm-like filomicelles, morphologically equivalent to filamentous viruses, have also been developed to exploit the long circulation and stealth-like properties of elongated nanoparticles. ${ }^{18}$ Likewise, avoidance of the monophagocytotic system (MPS) is a key advantage in using such technology. Opposed to the previous examples, where cellular interaction is avoided, nanoparticle size and shape are also essential features to improve effective surface interaction for immunostimulation. ${ }^{12,19}$

In order to control the morphological features of nanoparticles, a number of strategies have been developed, such as lithographic molding using the PRINT technology, ${ }^{20}$ mechanical polymer film-stretching ${ }^{21}$ and polymer-induced selfassembly (PISA), ${ }^{22,23}$ which are extensively reviewed elsewhere. $^{7,10,24}$ Recently, our group has demonstrated that the self-assembly of biodegradable block copolymers (BCPs) can

Special Issue: Biomacromolecules BPC

Received: August 15, 2018

Revised: September 22, 2018

Published: September 28, 2018 
provide a direct route toward the formation of polymersomal architectures that can be shape transformed into both oblate (discoidal or stomatosomal) and prolate (tubular) morphologies. $^{25,26}$ This was achieved through careful molecular engineering of the amphiphilic block copolymer poly(ethylene glycol)-block-poly(D,L-lactide) (PEG-PDLLA) and its assembly process. Parameters such as polymer chain length and thickness of the surface PEG-layer were varied in order to direct osmotically induced shape transformations toward stomatocytes $^{25}$ or nanotubes ${ }^{26}$ during dialysis of initially spherical polymersomes. Specially, biodegradable PEGPDLLA polymersomes with tubular morphology have great potential to provide a platform for nanomedicine applications. ${ }^{14,15}$ In particular, the engineering of these nanotubes into artificial antigen presenting cells that can trigger specific immune-activation is an exciting prospect. ${ }^{12,19,27,28}$ However, a greater degree of control must be achieved before such a system can be translated toward biomedical research due to the importance of systematic reproducibility and of uniformity in the aspect ratio of the resultant nanoparticles.

Here, we present the development of a robust approach for the controlled fabrication of well-defined nanotubes, based upon polymersomes with an average size of $\sim 100 \mathrm{~nm}$. After preparation of small uniform polymersomes via physical processing (extrusion), shape transformation into their prolate counterparts could be realized efficiently, generating truly nanotubular structures (Figure 1). Furthermore, using

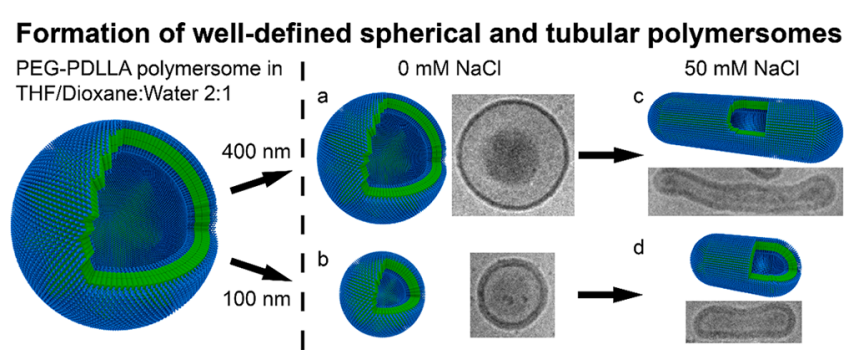

Figure 1. Schematic outlining of the extrusion of PEG-PDLLA polymersomes using (a) 400 and (b) $100 \mathrm{~nm}$ filters. Their osmotically induced shape transformation results in well-defined nanotubes (c) and $(d)$.

asymmetric flow field-flow fractionation (AF4) coupled with multiangle light scattering (MALS) we present a robust characterization methodology for such particles, where physical measurement of hydrodynamic radius $\left(R_{\mathrm{h}}\right)$ and radius of gyration $\left(R_{\mathrm{g}}\right)$ can be readily obtained and confirmed by cryotransmission electron microscopy (TEM). This technology now provides a robust platform for the generation of precision nanomedical formulations with control over both size and shape for applications such as immunotherapy and drug delivery.

\section{EXPERIMENTAL SECTION}

Materials. All chemicals were used as received unless otherwise stated. For the synthesis of poly(ethylene glycol)-b-poly(D,L-lactide) (PEG-PDLLA), poly(ethylene glycol) $1 \mathrm{~K}$ was purchased from JenKem technology and lyophilized before use. D,L-Lactide was purchased from Acros and used as supplied. DiD' Solid (DiIC18(5) solid (1,1'-dioctadecyl-3,3,3',3'-tetramethylindodicarbocyanine, 4chlorobenzenesulfonate salt) and Alexa Fluor 488 5-SDP Ester (Alexa Fluor 488 sulfodichlorophenol ester) were purchased from Thermo Fisher Scientific (Invitrogen). Albumin from bovine serum
(BSA) and sodium chloride were purchased from Merck. All other chemicals were supplied by Merck and were used without any purification. For the polymersome self-assembly and dialysis, ultrapure Milli-Q water was used, which was obtained from a Merck Millipore Q-Pod system (18.2 M $\Omega$ ) with a $0.22 \mu \mathrm{m}$ Millipore Express 40 filter. Dialysis membranes with a molecular weight cutoff (MWCO) of 12000-14000 Da from Spectra/Por were used for dialysis during the formation of polymersomes and nanotubes. Whatman Nuclepore track-etched membranes were purchased from Merck and used for polymersome extrusion together with $10 \mathrm{~mm}$ filter supports, purchased from Avanti Polar Lipids. Amicon Ultra 0.5 $\mathrm{mL}$ Centrifugal Filters $3 \mathrm{~K}$ were purchased from Merck and used for concentration of the polymersomes.

Instruments. Nuclear Magnetic Resonance (NMR). Proton nuclear magnetic resonance measurements were performed on a Bruker 400 Ultrashield spectrometer equipped with a Bruker SampleCase autosampler, using $\mathrm{CDCl}_{3}$ as a solvent and TMS as an internal standard.

Gel Permeation Chromatography (GPC). GPC was conducted using a Shimadzu Prominence-i GPC system with a PL gel $5 \mu \mathrm{m}$ mixed D and mixed C column (Polymer Laboratories) with PS standard and equipped with a Shimadzu RID-20A differential refractive index detector and THF used as an eluent with a flow rate of $1 \mathrm{~mL} \min ^{-1}$.

Dynamic Light Scattering (DLS). DLS measurements were performed on a Malvern instrument Zetasizer (model Nano ZSP) equipped with an auto sampler. Zetasizer software was used to process and analyze the data.

Cryogenic Transmission Electron Microscopy (cryo-TEM). Experiments were performed using a FEI Tecnai G2 Sphere $(200 \mathrm{kV}$ electron source) equipped with LaB6 filament utilizing a cryoholder or a FEI Titan ( $300 \mathrm{kV}$ electron source) equipped with autoloader station. Samples for cryo-TEM were prepared by treating the grids (Lacey carbon coated, R2/2, Cu, 200 mesh, EM sciences) in a Cressington 208 carbon coater for $40 \mathrm{~s}$. Then, $3 \mu \mathrm{L}$ of the polymersome solution was pipetted on the grid and blotted in a Vitrobot MARK III at $100 \%$ humidity. The grid was blotted for $3 \mathrm{~s}$ (offset -3 ) and directly plunged and frozen in liquid ethane. Processing of TEM images was performed with ImageJ, a program developed by $\mathrm{NIH}$ and available as public domain software at http:// rsbweb.nih.gov/ij/. The nanotube aspect ratio was calculated by dividing the measured length by the width of each tube and calculating the mean value.

Asymmetric Flow Field-Flow Fractionation and Multiangle Light Scattering (AF4-MALS). The Asymmetric Flow Field-Flow Fractionation-UV-QELS (AF4-UV-QELS) measurements were performed on a Wyatt Dualtec AF4 instrument connected to a Shimadzu LC2030 Prominence-i system with Shimadzu LC-2030 autosampler. The AF4 was connected to Wyatt Optilab rEX refractive index detector and Wyatt DAWN HELEOS II light scattering detector (MALS) installed at different angles $\left(12.9^{\circ}, 20.6^{\circ}, 29.6^{\circ}, 37.4^{\circ}, 44.8^{\circ}, 53.0^{\circ}\right.$, $61.1^{\circ}, 70.1^{\circ}, 80.1^{\circ}, 90.0^{\circ}, 99.9^{\circ}, 109.9^{\circ}, 120.1^{\circ}, 130.5^{\circ}, 149.1^{\circ}$, and $\left.157.8^{\circ}\right)$. The MALS laser operated at $658 \mathrm{~nm}$. Detectors were normalized using Bovine Serum Albumin (BSA). The processing and analysis of the light scattering data and radius of gyration $\left(R_{\mathrm{g}}\right)$ calculations were performed on Astra 7 software. As particles were of size $>50 \mathrm{~nm}$, the Berry model was used for analysis. ${ }^{29}$ All fractionations were performed on an AF4 short channel equipped with regenerated cellulose (RC), a $10 \mathrm{kDa}$ membrane (Millipore), and a spacer of $350 \mu \mathrm{m}$. The applied flow conditions are indicated in Table S1.

Confocal Microscopy. Confocal microscopy experiments were performed using the Leica TCS SP5X system with a HCX PL Apo CS $63 \times 1.20$ Water UV objective and HyD detector, scanning at a speed of $400 \mathrm{~Hz}$. For imaging of BSA-AF488-loaded polymersomes excitation at $490 \mathrm{~nm}$ and emission from 505 to $611.8 \mathrm{~nm}$ was used, along with a 1 airy pinhole. For imaging of DiD-loaded polymersomes excitation at $631 \mathrm{~nm}$ and emission from 650 to $750 \mathrm{~nm}$ was used, along with a 1 airy pinhole. 
Nanotube Purification via Size Exclusion Chromatography (SEC). SEC purification of the loaded polymersomes and nanotubes was conducted using a Shimadzu system (LC-20AD equipped with a SPD-20A photodiode array) with a Superose 6 column (GE Healthcare) using Milli-Q water as an eluent.

Fluorescence Spectroscopy. Fluorescence emission spectra of polymersomes loaded with fluorescent cargo were measured using the Cary Eclipse fluorescence spectrophotometer from Agilent. Diluted polymersome samples, obtained after their purification, were directly measured. The BSA-AF488 fluorescence emission spectrum was obtained by excitation at $460 \mathrm{~nm}$ using a $20 \mathrm{~nm}$ slit and emission was measured from $490 \mathrm{~nm}$ using a $20 \mathrm{~nm}$ slit. The DiD fluorescence emission spectrum was obtained by excitation at $600 \mathrm{~nm}$ using a 20 $\mathrm{nm}$ slit and emission was measured from $630 \mathrm{~nm}$ using a $10 \mathrm{~nm}$ slit.

\section{METHODS}

Preparation of PEG-PDLLA Polymersomes and Nanotubes. The synthesis of block copolymer $\mathrm{PEG}_{22}-\mathrm{PDLLA}_{45}$ was performed according to a previously described procedure (Supporting Information, section 1.1). In a $15 \mathrm{~mL}$ glass vial, $\mathrm{PEG}_{22}-\mathrm{PDLLA}_{45}$ $(20 \mathrm{mg}$ ) was weighed. To the block-co-polymer, $1.4 \mathrm{~mL}$ of dioxane and $0.6 \mathrm{~mL}$ of THF $(4: 1 \mathrm{v} / \mathrm{v})$ were added, and a magnetic stirrer bar was added to the solution. Subsequently, the vial was capped with a rubber septum and the solution was stirred for $30 \mathrm{~min}$. Using a syringe pump, $2 \mathrm{~mL}$ ( $50 \mathrm{vol} \%$ ) of Milli-Q water was added at a rate of $1 \mathrm{~mL} \mathrm{hr}^{-1}$. The obtained cloudy polymersome solution was directly transferred to a prehydrated dialysis membrane and dialysis was performed at $4{ }^{\circ} \mathrm{C}$ against precooled Milli-Q water $(1 \mathrm{~L})$ over $24 \mathrm{~h}$, with a water change after $1 \mathrm{~h}$. For the formation of nanotubes, the polymersome solution was dialyzed against a $50 \mathrm{mM}$ sodium chloride solution.

Fabrication of Well-Defined Spherical Polymersomes and Nanotubes. In order to form well-defined polymersomes, the preparation procedure was slightly adjusted. Prior to dialysis, the polymersome solution was extruded to obtain polymersomes with a well-defined size. To increase membrane flexibility for extrusion, the relative amount of organic solvent was therefore increased by only adding $1 \mathrm{~mL}$ (33 vol \%) of Milli-Q water at a rate of $1 \mathrm{~mL} \mathrm{hr}^{-1}$. The resulting polymersome solution was extruded by passing the solution 11 times through an Avanti Mini-Extruder, which was assembled with a $100 \mathrm{~nm}$ polycarbonate membrane filter supported by two $10 \mathrm{~mm}$ filter supports. The extruded polymersome solution was transferred to a prehydrated dialysis membrane. To preserve the spherical morphology of the resulting polymersomes, dialysis was performed at $4{ }^{\circ} \mathrm{C}$ against precooled Milli-Q water $(1 \mathrm{~L})$ over $24 \mathrm{~h}$ with a water change after $1 \mathrm{~h}$. Shape transformation into nanotubes was induced by immediate dialysis of the flexible extruded polymersome solution against a $50 \mathrm{mM}$ sodium chloride solution. Filters with membrane sizes of 200, 400, and $800 \mathrm{~nm}$ were used to prepare larger polymersomes.

Loading Polymersomes with Hydrophobic and Hydrophilic Cargos. Following the previously described method for polymersome formation, cargo-loaded polymersomes were formed. Polymersomes were loaded with hydrophobic cargo by initially mixing $\mathrm{DiD}$ with the copolymers prior to their assembly. Together with the copolymer, $\mathrm{DiD}$ was dissolved in an organic solvent mixture of dioxane and THF $(4: 1 \mathrm{v} / \mathrm{v})$ at a concentration of $0.5 \mathrm{mg} / \mathrm{mL}$. Of this solution, $2 \mathrm{~mL}$ was added to the weighed polymer, resulting in $0.5 \mathrm{wt} \% \mathrm{DiD}$ with respect to the polymer. Loading polymersomes with hydrophilic cargo was performed by adding $1 \mathrm{mg} / \mathrm{mL}$ BSA-AF488 in Milli-Q to the polymer solution. BSA was labeled with Alexa Fluor 488 according to the supplier's protocol (Supporting Information, section 1.2). Extrusion and dialysis against Milli-Q water or $50 \mathrm{mM} \mathrm{NaCl}$ solution (to induce tube formation) were performed as described above. After dialysis, unincorporated BSA-AF488 and DiD were removed using size exclusion chromatography (SEC). The eluate was concentrated using centrifugal filters ( $3 \mathrm{kDa}$ Amicon Ultra $0.5 \mathrm{~mL}$ ), according to the manufacturer's protocol (Merck), for $30 \mathrm{~min}$ at $4{ }^{\circ} \mathrm{C}$.

\section{RESULTS AND DISCUSSION}

According to our previous research, $\mathrm{PEG}_{22}-\mathrm{PDLLA}_{45} \mathrm{BCPs}$ are suitable for the formation of tubular polymersomes via an osmotically induced shape transformation protocol. ${ }^{26}$ Having synthesized the copolymer via ring-opening polymerization, catalyzed by 1,8 -diazabicyclo[5.4.0] undec-7-ene (DBU), the composition and polydispersity were calculated from the ${ }^{1} \mathrm{H}$ NMR and gel permeation chromatography (GPC) data, respectively $(\nexists=1.1$; Figures S1 and S2). Copolymer selfassembly into spherical polymersomes was promoted by addition of water $(50 \mathrm{vol} \%)$ to a BCP solution in a dioxane/THF mixture $(4: 1 \mathrm{v} / \mathrm{v})$. PEG-PDLLA polymersomes were subsequently dialyzed (against Milli-Q) to remove organic solvent, resulting in rigid spherical polymersomes with a diameter of approximately $500 \mathrm{~nm}$ (Figure S3a). When the dialysis procedure was performed on the flexible spherical polymersomes with solutions of increasing salt concentration, shape transformation into nanotubes was facilitated, with $[\mathrm{NaCl}]=50 \mathrm{mM}$ yielding nanotubes with a length of approximately $1 \mu \mathrm{m}$ (Figure S3b).

This shape transformation process occurs as the polymersomes are exposed to hypertonic conditions, which results in reduction of the internal volume (deflation), concomitant with the minimization of the bending energy in the polymeric membrane. Bending energy $E_{\mathrm{b}}$ can be described as a function of three parameters, the bending rigidity $k$, the mean surface curvature $C$, and the spontaneous curvature $C_{0}$ (eq 1$){ }^{26}$

$$
E_{\mathrm{b}}=\frac{k}{2} \oint\left(2 C-C_{0}\right)^{2} \mathrm{~d} A
$$

During the deflation of PEG-PDLLA spherical polymersomes, a determining factor for the formation of tubular morphologies is the slight positive contribution of spontaneous curvature $C_{0}$ (originating from asymmetry in the copolymer conformation between inner and outer surfaces), resulting in the active formation of prolates (tubes). ${ }^{26}$ Interestingly, the bending rigidity $k$ is strongly affected by the presence of organic solvent during the shape transformation process. As spherical polymersomes are dialyzed against water, the organic solvent (dioxane/THF at $4: 1 \mathrm{v} / \mathrm{v}$ ) is slowly removed. The significance of the organic solvent is thermodynamically important as it plasticizes the membrane and reduces $k$, which in turn reduces $E_{\mathrm{b}}$, so that the shape transformation can occur (if $E_{\mathrm{b}}$ was large enough to exceed the osmotic pressure, then no change would occur).

To develop biodegradable polymersomes with features that are relevant for nanomedical research, achieving control over both size and shape is of great importance. Utilizing the native flexibility of the polymersome membrane prior to dialysis, we explored the applicability of an extrusion methodology to facilitate greater size control in the fabrication of well-defined nanotubes with greater potential as a "precision" nanomedical platform.

To control the size of polymersomes, the nascent dispersion (with 50 vol \% water) was extruded using different membrane filters (pore size 100 and $400 \mathrm{~nm}$ ), followed by dialysis in water. Dynamic light scattering (DLS) showed that the size of the polymersomes decreased to about 120 (PDI 0.1) and $200 \mathrm{~nm}$ (PDI $\sim 0.2$ ), respectively, compared to $550 \mathrm{~nm}$ without extrusion (Figure S4). The morphology of the polymersomes was analyzed using cryo-TEM (Figure S5). Small monodisperse spheres of about $120 \mathrm{~nm}$ were observed 
for samples prepared using the $100 \mathrm{~nm}$ filter (Figure S5a) whereas a more polydisperse sample was obtained with the 400 $\mathrm{nm}$ filter (Figure S5b), in agreement with DLS data. To induce shape transformation, the extruded polymersomes were dialyzed against $50 \mathrm{mM} \mathrm{NaCl}$ solution, which did not show any remarkable change in size as compared to those dialyzed against water, according to DLS (Figure S4b). Indeed, cryoTEM showed that $120 \mathrm{~nm}$ polymersomes did not transform into tubes under these conditions; rather, small invaginations were observed (Figure S5c). For the $200 \mathrm{~nm}$ sample, a heterogeneous mixture of polymersomal morphologies was observed (Figure S5d). With this in mind, it was clear that, although well-defined polymersomes of $100 \mathrm{~nm}$ could be fabricated, shape transformation could not be induced. It was hypothesized that such small polymersomes, having a much higher surface curvature $(C)$, would require modulation of the conditions in order to overcome the increase in $E_{\mathrm{b}}$ that would prohibit shape transformation.

We, thus, optimized the conditions under which extruded polymersomes could be shape transformed, focusing on solvent composition and ionic strength during dialysis. As the organic solvent works as a plasticizing agent, it acts to reduce membrane rigidity $k$ and, thus, the bending energy. During the assembly process, it was observed that after addition of approximately $20 \mathrm{vol} \%$ of water the solution already turned cloudy, indicating PEG-PDLLA self-assembly. This demonstrated that it is not required to add $50 \mathrm{vol} \%$ of water to the sample to induce polymersome formation, leaving us with a window of operation between approximately 20 to 50 vol \% water for the dialysis procedure. It was found that $33 \mathrm{vol} \%$ of water was the threshold for reproducible formation of stable polymersomes. Therefore, in order to optimize the amount of organic solvent and, consequently, membrane flexibility, we used $33 \mathrm{vol} \%$ of water, as opposed to the $50 \mathrm{vol} \%$ used under standard conditions, for the assembly of PEG-PDLLA polymersomes. ${ }^{25,26}$ Indeed, under these conditions, extrusion was highly effective in resizing the polymersomes from about $500 \mathrm{~nm}$ down to $250($ PDI $\approx 0.2)$ or $100($ PDI $<0.1) \mathrm{nm}$ using the 400 or $100 \mathrm{~nm}$ extrusion filter membranes, respectively (Figures 2a,b and S6).

With an established protocol to fabricate polymersomes of $100 \mathrm{~nm}$, a size that is highly desirable for nanomedical applications due to favorable properties such as circulation time and tumor penetration, ${ }^{1,30}$ we investigated the shape transformation protocol to find suitable conditions to generate tubes. Osmotically induced shape transformation of PEGPDLLA nanotubes was attempted by dialyzing spherical polymersomes against $50 \mathrm{mM} \mathrm{NaCl}$ at $4{ }^{\circ} \mathrm{C}$ following extrusion. ${ }^{26}$ Although DLS did not indicate significant changes in $R_{\mathrm{h}}$ (Figure S6), it was clear from cryo-TEM that a structural change had occurred in both polymersome samples (Figure $2 \mathrm{c}, \mathrm{d})$. Although shape transformation of as-prepared polymersomes into $\approx 1 \mu \mathrm{m}$ nanotubes has been previously reported (Figure S3), the formation of these small $(\approx 100 \mathrm{~nm})$ nanotubes is unprecedented. Image analysis showed that extrusion of polymersomes using a $400 \mathrm{~nm}$ filter yielded structures with an average diameter of $213 \mathrm{~nm}$, whereas extrusion using a $100 \mathrm{~nm}$ filter resulted in polymersomes with average diameter of $82 \mathrm{~nm}$ (Figure 3a,b). Shape transformation of polymersomes extruded using 400 and $100 \mathrm{~nm}$ filters resulted in nanotubes with average dimensions (length $\times$ width) of $630 \times 85 \mathrm{~nm}$ and $137 \times 55 \mathrm{~nm}$ (Figures $3 \mathrm{c}$,d and
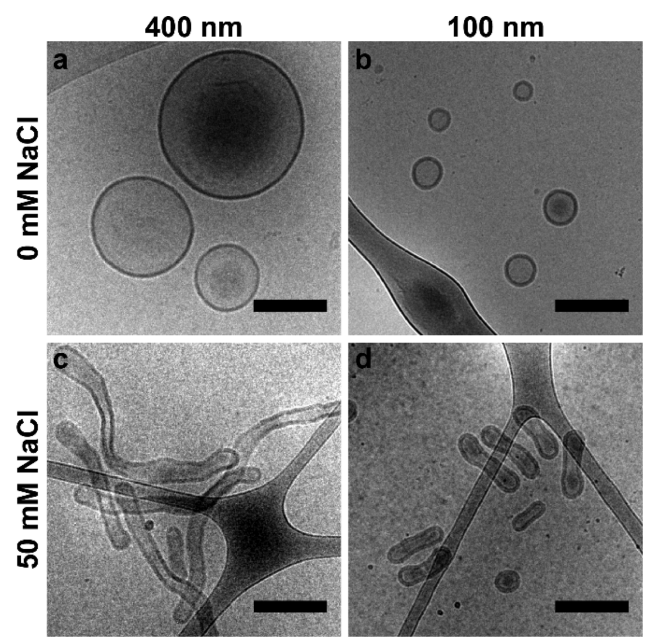

Figure 2. Cryo-TEM images of the extruded $\mathrm{PEG}_{22}-\mathrm{PDLLA}_{45}$ polymersomes using (a) $400 \mathrm{~nm}$ filter and (b) $100 \mathrm{~nm}$ filter, and (c, d) following their shape transformation into elongated nanotubes as a result of their exposure into a hypertonic shock facilitated by dialysis $($ scale bar $=250 \mathrm{~nm})$.

S7); their mean aspect ratios were determined at $9.8 \pm 9.7$ and $2.7 \pm 1.5$ respectively.

To investigate the effect of salt on the shape transformation of the $100 \mathrm{~nm}$ extruded polymersomes, four different concentrations were used $(0,10,50$, and $100 \mathrm{mM} \mathrm{NaCl})$ and analyzed using cryo-TEM (Figure 4 ). While for dialysis against 0 and $10 \mathrm{mM} \mathrm{NaCl}$ spheres were observed, dialysis against 50 or $100 \mathrm{mM}$ yielded nanotubes. These results indicate that there is a certain boundary osmotic pressure needed to induce polymersome deflation. For larger polymersomes, we have reported that shape transformation occurs when dialyzing against $10 \mathrm{mM} \mathrm{NaCl}$; the necessary higher salt concentration for the shape change process of the smaller polymersomes is (again) a consequence of increased surface curvature and larger $E_{\mathrm{b}}$ that needs to be overcome by the osmotic pressure.

In addition to DLS and cryo-TEM, the polymersome morphologies were analyzed using Asymmetric Flow FieldFlow Fractionation (AF4) coupled with static light scattering (MALS) and dynamic light scattering detectors (QELS). AF4 separation methods were optimized so that elution of various polymersomes could be observed in a size-dependent fashion, with the smallest polymersomes eluting first (Figure 5). Previously, we demonstrated that the combination of MALS and QELS provides quantitative information on particle morphology. ${ }^{29}$ From MALS data the radius of gyration $\left(R_{\mathrm{g}}\right)$ can be determined, which is the root-mean-square radius ( $\mathrm{rms}$ ) from the center of mass of the particle. With QELS, the hydrodynamic radius $R_{\mathrm{h}}$ can be determined. Simultaneous measurement of both $R_{\mathrm{g}}$ and $R_{\mathrm{h}}$ provides information on the particle aspect ratio, as described by the shape factor $\rho\left(=R_{\mathrm{g}} /\right.$ $\left.R_{\mathrm{h}}\right){ }^{31}$ In the case of spherical particles, $\rho$ has a value of $\sim 1$, while this value exceeds 1.3 for prolates (nanotubes) and becomes larger when their aspect ratio increases. Nonextruded polymersomes had an average $\rho$ value of 1 (Figure S8a). Regular nanotubes (obtained after dialysis of large polymersomes against $50 \mathrm{mM} \mathrm{NaCl}$ ), yielded an average $\rho$ value of 2.2 , highlighting their elongated form (Figure S8c). Extruded polymersomes $(100 \mathrm{~nm})$ did not demonstrate such a marked change in the $\rho$ value, with only a small increase from $\sim 1$ to 

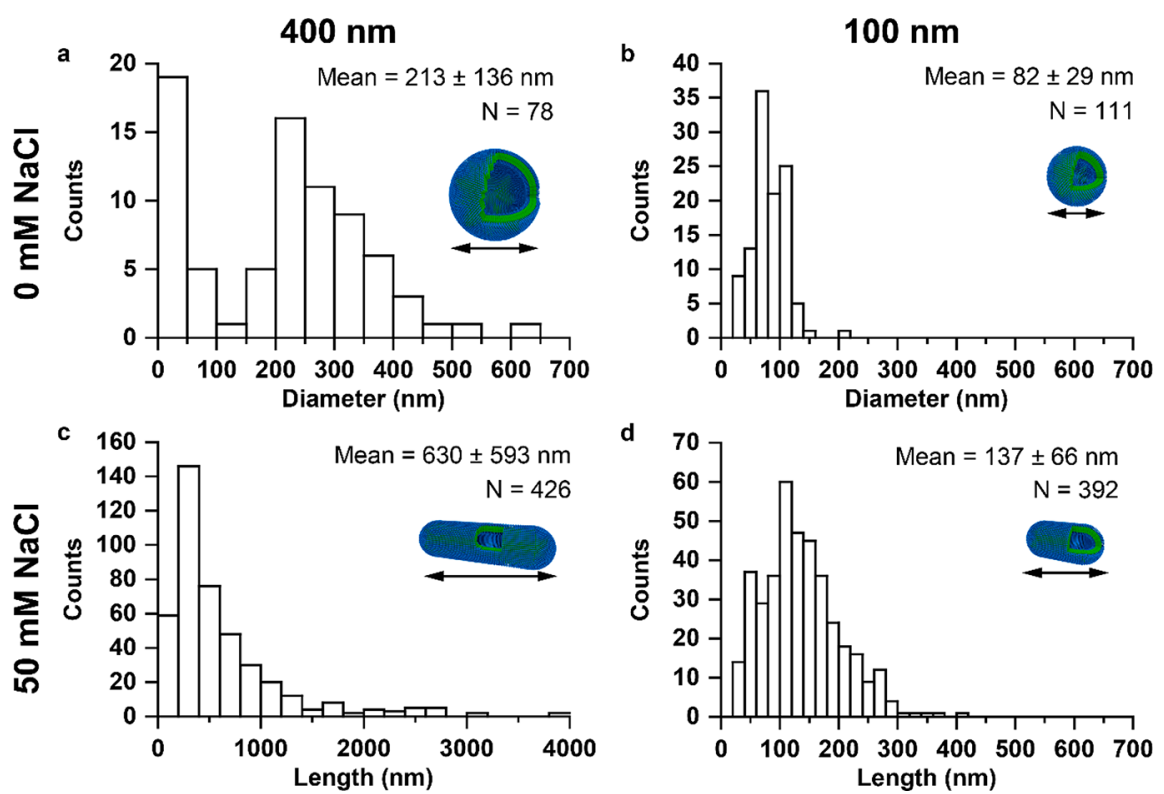

Figure 3. Histogram analysis of cryo-TEM data of extruded spherical polymersomes using (a) 400 and (b) $100 \mathrm{~nm}$ filters, and (c, d) after their shape transformation into nanotubes.

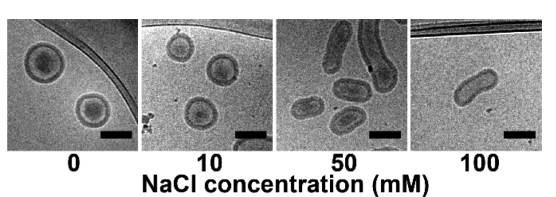

Figure 4. Salt concentration is essential for effective shape transformation of extruded polymersome spheres of $\sim 100 \mathrm{~nm}$ into nanotubes. Cryo-TEM analysis shows that dialysis in $10 \mathrm{mM} \mathrm{NaCl}$ is not sufficient to induce a shape-transformation, whereas tubular structures are formed when prepared with 50 and $100 \mathrm{mM} \mathrm{NaCl}$. Increasing $\mathrm{NaCl}$ concentration from 50 to $100 \mathrm{mM}$ does not result in any notable further elongation of the nanotubes. Scale bar $=100 \mathrm{~nm}$.

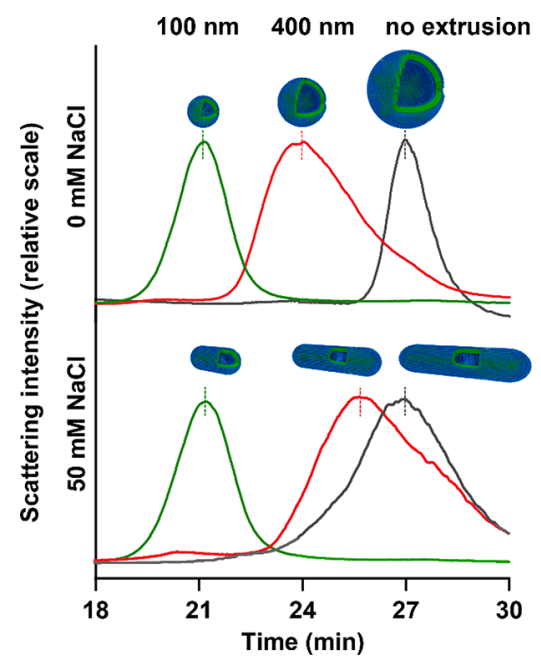

Figure 5. AF4-MALS-QELS shows different elution times of extruded polymersomes and their corresponding nanotubes.

1.2, highlighting their small aspect ratio (Figure $S 8 b, \mathrm{~d}$ ). This demonstrates that AF4-MALS/QELS, along with cryo-TEM, is a robust technique for validation of nanotubular structures. Our results indicate that not only size, but also the aspect ratio of biodegradable PEG-PDLLA polymersomes can be controlled.
As the engineering of such biodegradable polymeric architectures with discrete morphological characteristics is of great interest to future applications in nanomedicine, it is important to confirm their stability at physiological temperature. Therefore, stability was monitored using DLS before and after $24 \mathrm{~h}$ of incubation in Milli-Q at $37^{\circ} \mathrm{C}$ (Figure S9), which did not indicate any changes. To further demonstrate applicability for nanomedical applications, the possibility of loading proteins or small molecule (model) drugs is extremely important. In light of this, fluorescently labeled proteins (BSAAF488) and a hydrophobic dye (DiD) were simultaneously loaded in polymersomes during assembly. Under osmotic shock, polymersomes loaded with either hydrophobic or hydrophilic cargo were shape transformed into their tubular counter parts. Nanotubes were purified using size exclusion chromatography (Figure S10). AF4-UV-Light scattering was used to confirm the encapsulation. Polymersomes in which BSA-AF488 was encapsulated resulted in an absorbance peak at $488 \mathrm{~nm}$, characteristic to the AF488 covalently attached to BSA (Figure S11). Moreover, light scattering analysis of BSAfilled (spherical) polymersomes showed a reduction of the $R_{\mathrm{g}}$ values, yielding an average $R_{\mathrm{g}} / R_{\mathrm{h}}$ of $0.77 \pm 0.09$ (Figure S12), indicating the presence of BSA inside the polymersomes. ${ }^{32}$ Confocal microscopy and fluorescence spectroscopy confirmed successful loading of both dyes (Figure 6), although determination of colocalization was challenging due to the fast Brownian motion of the small polymersomes.

\section{CONCLUSION}

In summary, we have developed a robust methodology to generate small-sized biodegradable polymersomes through extrusion. By fine-tuning of the self-assembly, extrusion and shape transformation processes, tubular polymersomes with a size of approximately $100 \mathrm{~nm}$ were prepared. Such control over morphological characteristics is highly desirable for nanomedical applications such as therapeutic drug delivery and for specific manipulation of immune responses to battle cancer or autoimmune disease. Uncovering the physical basis of the underlying mechanism of shape transformation provided a 


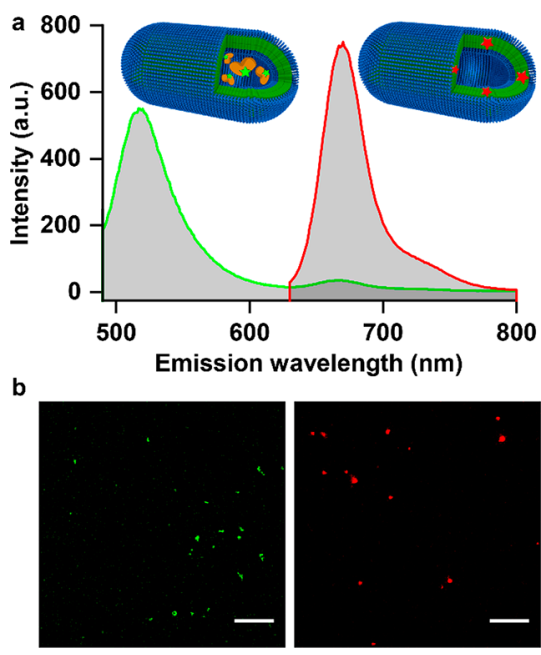

Figure 6. Fluorescence measurements of nanotubes loaded with BSAAF488 (green) and DiD (red). (a) Fluorescence emission spectra of loaded nanotubes show emission maxima around 525 and $665 \mathrm{~nm}$ as expected for these dyes. (b) Confocal microscopy shows fluorescent nanotubes. Scale bar $=1 \mu \mathrm{m}$.

direction on how such a well-controlled shape transformation of small polymersomes (with high bending energy) could be achieved. Moreover, we showed the possibility to load the resulting nanotubes with hydrophilic and hydrophobic cargoes, an important development of this system toward biomedical applications.

\section{ASSOCIATED CONTENT}

\section{S Supporting Information}

The Supporting Information is available free of charge on the ACS Publications website at DOI: 10.1021/acs.biomac. 8 b01245.

Copolymer characterization, cryo-TEM data analysis, and MALS and DLS data analysis (PDF).

\section{AUTHOR INFORMATION}

\section{Corresponding Authors}

*E-mail: j.c.m.v.hest@tue.nl.

*E-mail: 1.k.e.a.abdelmohsen@tue.nl.

\section{ORCID $\odot$}

Alexander F. Mason: 0000-0002-2847-0253

David S. Williams: 0000-0002-8209-6899

Loai K. E. A. Abdelmohsen: 0000-0002-0094-1893

Jan C.M. van Hest: 0000-0001-7973-2404

Notes

The authors declare no competing financial interest.

\section{ACKNOWLEDGMENTS}

The authors would like to thank Bastiaan Buddingh' for assistance with confocal microscopy. The authors would like to acknowledge the ERC Advanced Grant Artisym 694120, the Dutch Ministry of Education, Culture and Science (Gravitation Program 024.001.035) and NWO-NSFC Advanced Materials (Project 792.001.015) for funding. We thank the Ser Cymru II programme for support of DSW; this project received funding from the European Union's Horizon 2020 research and innovation programme under the Marie Skłodowska-Curie Grant Agreement No. 663830.

\section{REFERENCES}

(1) Petros, R. A.; Desimone, J. M. Strategies in the Design of Nanoparticles for Therapeutic Applications. Nat. Rev. Drug Discovery 2010, 9 (8), 615-627.

(2) Williams, D. S.; Pijpers, I. A. B.; Ridolfo, R.; van Hest, J. C. M. Controlling the Morphology of Copolymeric Vectors for next Generation Nanomedicine. J. Controlled Release 2017, 259, 29-39.

(3) Lammers, T.; Kiessling, F.; Hennink, W. E.; Storm, G. Drug Targeting to Tumors: Principles, Pitfalls and (Pre-) Clinical Progress. J. Controlled Release 2012, 161 (2), 175-187.

(4) Meng, F.; Zhong, Z.; Feijen, J. Stimuli-Responsive Polymersomes for Programmed Drug Delivery. Biomacromolecules 2009, 10 (2), 197-209.

(5) Wibroe, P. P.; Anselmo, A. C.; Nilsson, P. H.; Sarode, A.; Gupta, V.; Urbanics, R.; Szebeni, J.; Hunter, A. C.; Mitragotri, S.; Mollnes, T. E.; Moghimi, S. M. Bypassing Adverse Injection Reactions to Nanoparticles through Shape Modification and Attachment to Erythrocytes. Nat. Nanotechnol. 2017, 12 (6), 589-594.

(6) Geng, Y.; Dalhaimer, P.; Cai, S.; Tsai, R.; Tewari, M.; Minko, T.; Discher, D. E. Shape Effects of Filaments versus Spherical Particles in Flow and Drug Delivery. Nat. Nanotechnol. 2007, 2 (4), 249-255.

(7) Mitragotri, S.; Lahann, J. Physical Approaches to Biomaterial Design. Nat. Mater. 2009, 8 (1), 15-23.

(8) Duan, X.; Li, Y. Physicochemical Characteristics of Nanoparticles Affect Circulation, Biodistribution, Cellular Internalization, and Trafficking. Small 2013, 9 (9-10), 1521-1532.

(9) Decuzzi, P.; Godin, B.; Tanaka, T.; Lee, S. Y.; Chiappini, C.; Liu, X.; Ferrari, M. Size and Shape Effects in the Biodistribution of Intravascularly Injected Particles. J. Controlled Release 2010, 141 (3), $320-327$.

(10) Champion, J. A.; Mitragotri, S. Role of Target Geometry in Phagocytosis. Proc. Natl. Acad. Sci. U. S. A. 2006, 103 (13), 49304934.

(11) Massignani, M.; Lopresti, C.; Blanazs, A.; Madsen, J.; Armes, S. P.; Lewis, A. L.; Battaglia, G. Controlling Cellular Uptake by Surface Chemistry, Size, and Surface Topology at the Nanoscale. Small 2009, 5 (21), 2424-2432.

(12) Hotaling, N. a.; Tang, L.; Irvine, D. J.; Babensee, J. E. Biomaterial Strategies for Immunomodulation. Annu. Rev. Biomed. Eng. 2015, 17 (1), 317-349.

(13) Champion, J. A.; Katare, Y. K.; Mitragotri, S. Particle Shape: A New Design Parameter for Micro- and Nanoscale Drug Delivery Carriers. J. Controlled Release 2007, 121 (1-2), 3-9.

(14) Yealland, G.; Battaglia, G.; Bandmann, O.; Mortiboys, H. Rescue of Mitochondrial Function in Parkin-Mutant Fibroblasts Using Drug Loaded PMPC-PDPA Polymersomes and Tubular Polymersomes. Neurosci. Lett. 2016, 630, 23-29.

(15) Robertson, J. D.; Yealland, G.; Avila-Olias, M.; Chierico, L.; Bandmann, O.; Renshaw, S. A.; Battaglia, G. PH-Sensitive Tubular Polymersomes: Formation and Applications in Cellular Delivery. ACS Nano 2014, 8 (5), 4650-4661.

(16) Wong, C. K.; Mason, A. F.; Stenzel, M. H.; Thordarson, P. Formation of Non-Spherical Polymersomes Driven by Hydrophobic Directional Aromatic Perylene Interactions. Nat. Commun. 2017, 8 (1), 1240.

(17) Lim, S. K.; Wong, A. S. W.; De Hoog, H. P. M.; Rangamani, P.; Parikh, A. N.; Nallani, M.; Sandin, S.; Liedberg, B. Spontaneous Formation of Nanometer Scale Tubular Vesicles in Aqueous Mixtures of Lipid and Block Copolymer Amphiphiles. Soft Matter 2017, 13 (6), $1107-1115$.

(18) Geng, Y.; Dalhaimer, P.; Cai, S.; Tsai, R.; Tewari, M.; Minko, T.; Discher, D. E. Shape Effects of Filaments versus Spherical Particles in Flow and Drug Delivery. Nat. Nanotechnol. 2007, 2 (4), 249-255.

(19) Hickey, J. W.; Vicente, F. P.; Howard, G. P.; Mao, H.-Q.; Schneck, J. P. Biologically Inspired Design of Nanoparticle Artificial Antigen-Presenting Cells for Immunomodulation. Nano Lett. 2017, 17 (11), 7045-7054. 
(20) DeSimone, J. M. Co-Opting Moore's Law: Therapeutics, Vaccines and Interfacially Active Particles Manufactured via PRINT®. J. Controlled Release 2016, 240, 541-543.

(21) Champion, J. A.; Katare, Y. K.; Mitragotri, S. Making Polymeric Micro- and Nanoparticles of Complex Shapes. Proc. Natl. Acad. Sci. U. S. A. 2007, 104 (29), 11901-11904.

(22) Salva, R.; Le Meins, J. F.; Sandre, O.; Bruîlet, A.; Schmutz, M.; Guenoun, P.; Lecommandoux, S. Polymersome Shape Transformation at the Nanoscale. ACS Nano 2013, 7 (10), 9298-9311.

(23) Ratcliffe, L. P. D.; McKenzie, B. E.; Le Bouëdec, G. M. D.; Williams, C. N.; Brown, S. L.; Armes, S. P. Polymerization-Induced Self-Assembly of All-Acrylic Diblock Copolymers via RAFT Dispersion Polymerization in Alkanes. Macromolecules 2015, 48 (23), 8594-8607.

(24) Zhang, P.; Xia, J.; Luo, S. Generation of Well-Defined Micro/ Nanoparticles via Advanced Manufacturing Techniques for Therapeutic Delivery. Materials 2018, 11 (4), 623.

(25) Pijpers, I. A. B.; Abdelmohsen, L. K. E. A.; Williams, D. S.; van Hest, J. C. M. Morphology Under Control: Engineering Biodegradable Stomatocytes. ACS Macro Lett. 2017, 6, 1217-1222.

(26) Abdelmohsen, L. K. E. A.; Williams, D. S.; Pille, J.; Ozel, S. G.; Rikken, R. S. M.; Wilson, D. A.; Van Hest, J. C. M. Formation of Well-Defined, Functional Nanotubes via Osmotically Induced Shape Transformation of Biodegradable Polymersomes. J. Am. Chem. Soc. 2016, 138 (30), 9353-9356.

(27) Hammink, R.; Mandal, S.; Eggermont, L. J.; Nooteboom, M.; Willems, P. H. G. M.; Tel, J.; Rowan, A. E.; Figdor, C. G.; Blank, K. G. Controlling T-Cell Activation with Synthetic Dendritic Cells Using the Multivalency Effect. ACS Omega 2017, 2 (3), 937-945.

(28) Meyer, R. A.; Sunshine, J. C.; Perica, K.; Kosmides, A. K.; Aje, K.; Schneck, J. P.; Green, J. J. Biodegradable Nanoellipsoidal Artificial Antigen Presenting Cells for Antigen Specific T-Cell Activation. Small 2015, 11 (13), 1519-1525.

(29) Abdelmohsen, L. K. E. A.; Rikken, R. S. M.; Christianen, P. C. M.; van Hest, J. C. M.; Wilson, D. A. Shape Characterization of Polymersome Morphologies via Light Scattering Techniques. Polymer 2016, 107, 445-449.

(30) Farokhzad, O. C.; Langer, R. Impact of Nanotechnology on Drug on Delivery. ACS Nano 2009, 3 (1), 16-20.

(31) Boye, S.; Ennen, F.; Scharfenberg, L.; Appelhans, D.; Nilsson, L.; Lederer, A. From 1D Rods to 3D Networks: A Biohybrid Topological Diversity Investigated by Asymmetrical Flow Field-Flow Fractionation. Macromolecules 2015, 48 (13), 4607-4619.

(32) Stauch, O.; Schubert, R.; Savin, G.; Burchard, W. Structure of Artificial Cytoskeleton Containing Liposomes in Aqueous Solution Studied by Static and Dynamic Light Scattering. Biomacromolecules 2002, 3, 565-578. 